Andrew S. Tseng, MD, MPH

Department of Cardiovascular Diseases,

Mayo Clinic, Rochester, MN
Fadi E. Shamoun, MD

Department of Cardiovascular Diseases, Mayo Clinic, Phoenix, AZ
Lisa A. Marks, MLS

Mayo Clinic Library Services,

Mayo Clinic, Phoenix, AZ
Neera Agrwal, MD, PhD

Department of Internal Medicine,

Mayo Clinic, Phoenix, AZ

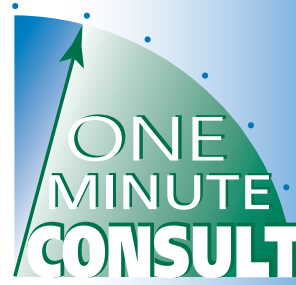

BRIEF ANSWERS

TO SPECIFIC

CLINICAL

QUESTIONS

\section{: What antithrombotic therapy should I use for my patient with atrial fibrillation who underwent percutaneous coronary inter- vention or had an acute coronary syndrome?}

coronary syndrome or percutaneous coronary intervention).

Both atrial fibrillation and coronary artery disease are common in the elderly, they share important risk factors, and they often occur concomitantly. Before direct oral anticoagulants were developed, patients with atrial fibrillation were often given triple therapy after experiencing acute coronary syndromes or undergoing percutaneous coronary interventions. This consisted of a vitamin $\mathrm{K}$ antagonist (eg, warfarin) and dual antiplatelet therapy. While oral anticoagulation and antiplatelet therapy separately increase the risk of bleeding, the risk is even higher when they are combined, and higher still with triple therapy. ${ }^{3}$

\section{DOUBLE VS TRIPLE THERAPY}

The safety and efficacy of double vs triple therapy has been evaluated in several randomized controlled trials. ${ }^{4-8}$

The WOEST trial ${ }^{4}$ (What Is the Optimal Antiplatelet and Anticoagulant Therapy in Patients With Oral Anticoagulation and Coronary Stenting?) compared double vs triple therapy, with a vitamin $\mathrm{K}$ antagonist as the anticoagulant, in patients with atrial fibrillation requiring percutaneous coronary intervention. It found that double therapy was safer and more effective than triple therapy, as measured at the end of 1 year.

Other trials ${ }^{5-8}$ subsequently showed that double therapy using a direct oral anticoagulant posed a lower risk of bleeding and was not inferior in efficacy (in general, a composite
The risk of thrombosis should be balanced with the risk of bleeding 
outcome of mortality and ischemic events) compared with triple therapy using a vitamin $\mathrm{K}$ antagonist as the oral anticoagulant.

Gargiulo et $\mathrm{al},{ }^{9}$ in a meta-analysis of these trials, ${ }^{5-8}$ found that double therapy posed a lower risk of bleeding, defined as International Society on Thrombosis and Haemostasis major or clinically relevant nonmajor bleeding (risk ratio 0.62, 95\% confidence interval [CI] $0.47-0.81$, number needed to treat 14$)$. However, the trials were not specifically powered to detect a difference in ischemic event rates. While there was no difference in the rates of all-cause mortality, cardiovascular mortality, or trial-defined major adverse cardiac events, there was a small but significant increase in the risk of stent thrombosis with double therapy (risk ratio $1.59,95 \%$ CI 1.01-2.50, number needed to harm 273). ${ }^{9}$

\section{DIRECT ORAL ANTICOAGULANTS VS VITAMIN K ANTAGONISTS}

Comparing the type of anticoagulant, direct oral anticoagulant therapy was at least noninferior to vitamin $\mathrm{K}$ antagonist therapy in regard to bleeding outcomes, and noninferior in
For most

patients, double therapy

(an oral anticoagulant and a P2Y12 inhibitor) is reasonable regard to ischemic outcomes. ${ }^{5-8}$ The numbers needed to treat with a direct oral anticoagulant to prevent 1 bleeding event from a vitamin $\mathrm{K}$ antagonist ranged from 10 to 24 . There were no significant differences in ischemic outcomes between the 2 types of anticoagulants. However, as we said, these trials were not specifically powered to detect differences in ischemic events; the primary outcome of interest was bleeding.

\section{CURRENT GUIDELINES}

Current ACC/AHA/HRS guidelines ${ }^{1}$ recommend anticoagulation in atrial fibrillation if the $\mathrm{CHA}_{2} \mathrm{DS}_{2}$-VASc score is 2 or higher in men and 3 or higher in women. (To calculate the $\mathrm{CHA}_{2} \mathrm{DS}_{2}$-VASc score, 1 point each is given for congestive heart failure, hypertension, age greater than 65 [or 2 points for age $>75$ ], diabetes, stroke [2 points], vascular disease, and female sex category, for a maximum of 9 points.)

First-line oral anticoagulation therapy has traditionally consisted of a vitamin $\mathrm{K}$ antagonist but now includes the direct oral antico- agulants, such as dabigatran, rivaroxaban, apixaban, and edoxaban, ${ }^{1}$ which have become the preferred agents due to safety data.

For patients with atrial fibrillation requiring combined antithrombotic therapy for acute coronary syndromes or percutaneous coronary intervention, the ACC/AHA/ HRS, ${ }^{1}$ American College of Chest Physicians (ACCP),${ }^{10}$ and $\mathrm{ESC}^{2}$ guidelines recommend double or short-term triple therapy with an oral anticoagulant (vitamin $\mathrm{K}$ antagonist, rivaroxaban, or dabigatran) and clopidogrel, tailored on the basis of thrombotic risk and bleeding risk (discussed further below).

The ESC guidelines ${ }^{2}$ specifically recommend against the combination of a direct oral anticoagulant plus prasugrel or ticagrelor, given a lack of evidence and potential for increased bleeding based on registry data. The ACC/AHA/HRS guidelines ${ }^{1}$ allow ticagrelor as an alternative to clopidogrel in dual therapy with a vitamin $\mathrm{K}$ antagonist but not a direct oral anticoagulant.

The ACCP guidelines ${ }^{10}$ further recommend that direct oral anticoagulants be used at licensed dosing levels, particularly important with rivaroxaban and dabigatran, which were given in lower, nonapproved doses in their respective clinical trials. 5,6

The current guidelines regarding direct oral anticoagulants do not include apixaban or edoxaban. However, based on recent trials of these newer agents, ${ }^{7,8}$ double therapy with apixaban or edoxaban and clopidogrel may soon be formally recommended.

\section{PATIENTS AT HIGH THROMBOTIC RISK}

In their 2017 guidelines, ${ }^{2}$ the ESC recommended triple therapy for at least 1 month and up to 6 months in the subset of patients who underwent percutaneous coronary intervention or who had a high thrombotic risk. The $\mathrm{ESC}^{2}$ and $\mathrm{ACCP}^{10}$ guidelines enumerate these risk factors, which include:

- Prior stent thrombosis while receiving antiplatelet therapy

- Stenting of the last remaining patent coronary artery

- Diffuse multivessel disease, especially in patients with diabetes

- Chronic kidney disease 
- At least 3 stents implanted

- At least 3 lesions treated

- Bifurcation with 2 stents implanted

- Total stent length greater than $60 \mathrm{~mm}$

- Treatment of a chronic total occlusion

- Left main stenting.

\section{PATIENTS AT HIGH BLEEDING RISK}

The risk of thrombotic events should be balanced with the risk of bleeding. The most commonly used tool for assessing bleeding risk is the HAS-BLED score, in which points are given for hypertension, abnormal kidney or liver function, stroke, bleeding, labile international normalized ratio, elderly status, and use of drugs that predispose to bleeding or use of alcohol. A HAS-BLED score of 3 or more indicates a higher risk of bleeding.

The 2018 ACCP atrial fibrillation guidelines ${ }^{10}$ recommend using the HAS-BLED score, particularly in patients taking vitamin $\mathrm{K}$ antagonists, to tailor discussion of risk of bleeding while on anticoagulant therapy. While rarely a reason to avoid anticoagulation, a high HAS-BLED score should prompt clinicians to aggressively treat the modifiable aspects of the score to reduce the risk of bleeding. ${ }^{10}$ This is particularly important to consider when additional antiplatelet therapy is required after percutaneous coronary intervention.

\section{DURATION OF COMBINED ANTITHROMBOTIC THERAPY}

The overall duration of antiplatelet therapy is based on the indication for it (stable ischemic heart disease with percutaneous coronary intervention, acute coronary syndrome, and type of stent used). Patients who have stable ischemic heart disease who undergo percutaneous coronary intervention with bare-metal stents require at least 1 month of antiplatelet therapy. Those who receive drug-eluting stents require at least 3 months of antiplatelet therapy if they have a high risk of bleeding; otherwise, 6 months is preferred. In those with acute coronary syndrome with or without percutaneous intervention, antiplatelet therapy is recommended for at least 6 months if there is a high risk of bleeding; otherwise, 12 months is preferred. ${ }^{11}$
Whether to start patients on triple therapy and its duration is another consideration. The duration of initial triple therapy is tailored on the basis of individual thrombotic and ischemic risk, and the guidelines ${ }^{1,2,10}$ offer multiple strategies. In summary:

- For patients at low thrombotic risk, double therapy alone (ACC/AHA/HRS $)^{1}$ or 1 to 6 months of triple therapy $\left(\mathrm{ACCP}^{10}\right.$ and ESC $^{2}$ ) can be considered, with shorter durations of triple therapy if bleeding risk is high (HAS-BLED score $\geq 3$ ).

- For those at high thrombotic risk, all guidelines recommend triple therapy for 1 to 6 months, again tailored to bleeding risk. ${ }^{1,2,10}$

\section{TAKE-HOME MESSAGES}

Combined antithrombotic therapy in patients with atrial fibrillation with acute coronary syndrome or percutaneous coronary intervention requires a balanced consideration of bleeding risk vs ischemic risk.

For most patients with average ischemic risk, double therapy with a vitamin $\mathrm{K}$ antagonist or a direct oral anticoagulant-specifically rivaroxaban, dabigatran, and, probably soon, apixaban and edoxaban - with clopidogrel is reasonable. Direct oral anticoagulants, at licensed dosing, have become the preferred agents for many patients due to the lower risk of bleeding.

For patients at increased ischemic risk and average or lower bleeding risk, triple therapy for 1 to 6 months may be considered. The duration of triple therapy is tailored to the risk of bleeding, with a shorter duration if bleeding risk is high (eg, HAS-BLED score $\geq 3$, recent bleeding).

Given the current evidence, clopidogrel should be used in combined antithrombotic therapy. Ticagrelor can be considered for double therapy with a vitamin $\mathrm{K}$ antagonist.

This is a complex and evolving field, and as new evidence comes out and technology improves, our practice of using combined antithrombotic therapies in these high-risk patients will undoubtedly continue to change.

\section{DISCLOSURES}

The authors report no relevant financial relationships which, in the context of their contributions, could be perceived as a potential conflict of interest. 


\section{ANTITHROMBOTIC THERAPY IN ATRIAL FIBRILLATION}

\section{REFERENCES}

1. January CT, Wann LS, Alpert JS, et al. 2014 AHA/ACC/HRS guideline for the management of patients with atrial fibrillation: a report of the American College of Cardiology/American Heart Association Task Force on Practice Guidelines and the Heart Rhythm Society. J Am Coll Cardiol 2014; 64(21):e1-e76. doi:10.1016/j.jacc.2014.03.022

2. Valgimigli M, Bueno H, Byrne RA, et al. 2017 ESC focused update on dual antiplatelet therapy in coronary artery disease developed in collaboration with EACTS: the Task Force for dual antiplatelet therapy in coronary artery disease of the European Society of Cardiology (ESC) and of the European Association for Cardio-Thoracic Surgery (EACTS). Eur Heart J 2018; 39(3):213-260. doi:10.1093/eurheartj/ehx419

3. Lamberts M, Gislason GH, Olesen BJ, et al. Oral anticoagulation and antiplatelets in atrial fibrillation patients after myocardial infarction and coronary intervention. J Am Coll Cardiol 2013; 62(11): 981-989. doi:10.1016/j.jacc.2013.05.029

4. Dewilde WJ, Oirbans T, Verheugt FW, et al. Use of clopidogrel with or without aspirin in patients taking oral anticoagulant therapy and undergoing percutaneous coronary intervention: an open-label, randomised, controlled trial. Lancet 2013; 381(9872):1107-1115. doi:10.1016/S0140-6736(12)62177-1

5. Cannon CP, Bhatt DL,Oldgren J, et al. Dual antithrombotic therapy with dabigatran after PCI in atrial fibrillation. N Engl J Med 2017; 377:1513-1524. doi:10.1056/NEJMoa1708454

6. Gibson CM, Mehran R, Bode C, et al. Prevention of bleeding in patients with atrial fibrillation undergoing PCl. N Engl J Med 2016; 375(25):2423-2434. doi:10.1056/NEJMoa1611594
7. Lopes RD, Heizer G, Aronson R, et al. Antithrombotic therapy after acute coronary syndrome or $\mathrm{PCl}$ in atrial fibrillation. $\mathrm{N}$ Engl J Med 2019; 380(16):1509-1524. doi:10.1056/NEJMoa1817083

8. Vranckx $P$, Valgimigli $M$, Eckardt $L$, et al. Edoxaban-based versus vitamin $\mathrm{K}$ antagonist-based antithrombotic regimen after successful coronary stenting in patients with atrial fibrillation (ENTRUSTAF PCI): a randomised, open-label, phase $3 \mathrm{~b}$ trial. Lancet 2019; 394(10206):1335-1343. doi:10.1016/S0140-6736(19)31872-0

9. Gargiulo G, Goette A, Tijssen J, et al. Safety and efficacy outcomes of double vs. triple antithrombotic therapy in patients with atrial fibrillation following percutaneous coronary intervention: a systematic review and meta-analysis of non-vitamin $\mathrm{K}$ antagonist oral anticoagulant-based randomized clinical trials. Eur Heart J; 2019: 40(46): 3757-3767. doi:10.1093/eurheartj/ehz732

10. Lip GYH, Banerjee A, Boriani G, et al. Antithrombotic therapy for atrial fibrillation: Chest guideline and expert panel report. Chest 2018; 154:1121-1201. doi:10.1016/j.chest.2018.07.040

11. Levine GN, Bates ER, Bittl JA, et al. 2016 ACC/AHA guideline focused update on duration of dual antiplatelet therapy in patients with coronary artery disease: a report of the American College of Cardiology/American Heart Association Task Force on Clinical Practice Guidelines. J Thorac Cardiovasc Surg 2016; 152(5):1243-1275. doi:10.1016/j.jtcvs.2016.07.044

Address: Neera Agrwal, MD, PhD, Mayo Clinic, 5777 East Mayo Boulevard, Phoenix, AZ 85054; agrwal.neera@mayo.edu 